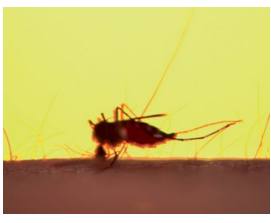

Malaria vaccine sets a record p786

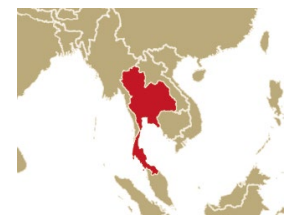

\begin{abstract}
Biotech news from around the world p788
\end{abstract}

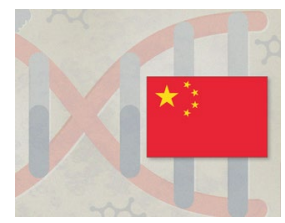
China gains global sway p789

\title{
'Super-antibodies' could curb COVID-19 and help avert future pandemics
}

\author{
Companies are designing next-generation antibodies modeled on those taken from unique individuals whose \\ immune systems can neutralize any COVID-19 variant-and related coronaviruses, too.
}

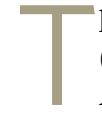

he US Food and Drug Administration (FDA) granted Emergency Use Authorization (EUA) in late May to sotrovimab, providing a new therapeutic weapon in the fight against SARS-CoV-2and future coronaviruses with pandemic potential.

According to analysts and researchers alike, so-called super-antibodies such as sotrovimab should have an edge over first-generation monoclonal antibody (mAb) therapies for COVID-19 because of their broad neutralization capacity in the face of emerging virus variants. "Physicians aren't going to sequence what version of the virus people have, so they'll go for the antibodies that have the higher barrier to resistance or the ones that work on [known] variants," says Phil Nadeau, an analyst at Cowen.

The antibody therapy from Vir Biotechnology and GlaxoSmithKline, a recombinant human immunoglobulin $\mathrm{G} 1 \mathrm{mAb}$, is now the third mAb-based treatment marketed for individuals with mild-to-moderate COVID-19 who are at high risk for progression to severe disease. (Eli Lilly and Regeneron each have a two-mAb cocktail with EUAs for the same indication.) And although sales opportunities should diminish for all these products as vaccination rates increase worldwide, Nadeau anticipates there will be a sustained market for COVID-19 mAbs to help treat individuals who, for medical reasons, can't mount an appropriate immune response to vaccination or, for whatever reason, elect not to get the shot.

According to his models, sotrovimab should capture around $10 \%$ of the $\$ 3$ billion global COVID-19 mAb market this year, rising to $30 \%$ of the $\$ 1.67$ billion market in 2022.

Other broadly cross-reactive mAbs could soon be jockeying for market share as well,

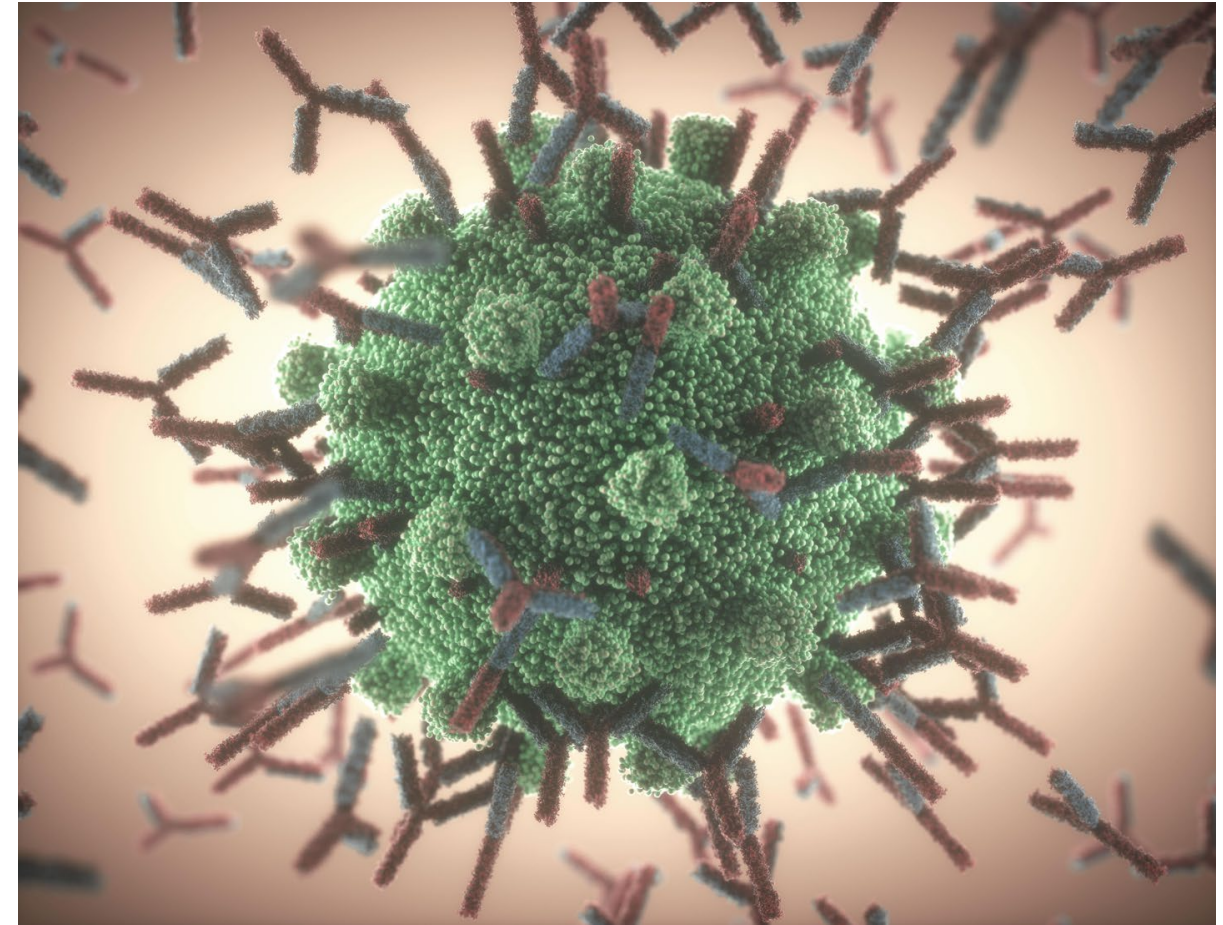

Antibodies attacking a coronavirus (artist's impression). Super-antibodies that can neutralize even the most worrying versions of SARS-CoV-2 could be used both to prevent and to treat infections. Credit: Kiyoshi Takahase Segundo / Alamy Stock Photo

as investment money flows in to rush some of the leading candidates through late-stage clinical development. In April, for example, Adagio Therapeutics raised $\$ 336$ million (on top of the $\$ 130$ million war chest it amassed last year) to bankroll large-scale trials of ADG20, an mAb now being evaluated for use as a therapy and for prevention. Startups such as Centivax, Corat Therapeutics, IDBiologics, Leyden Labs, Memo Therapeutics and SpikImm are working on next-generation mAbs for COVID-19 as well.
Sotrovimab traces its roots back to blood drawn in 2013 from an individual who had recovered from the 2003 outbreak of severe acute respiratory syndrome (SARS); ADG20 has a similar origin story, while most other clinical-stage mAbs were inspired by antibodies found in more recent COVID-19 survivors. Many companies then optimized their mAbs by extending half-lives, enhancing neutralizing activity, manipulating constant region $(\mathrm{Fc})$-mediated effector functions, 


\section{Novavax's fridge-friendly vaccine impresses}

Novavax has unveiled results from a large clinical trial showing that its vaccine is $90.4 \%$ effective in preventing symptomatic COVID-19 and $100 \%$ protective against moderate and severe disease. Results from the phase 3 PREVENT-19 trial with 30,000 participants across the United States and Mexico had been eagerly awaited for the vaccine's presumed advantages over existing jabs. Unlike the approved Pfizer, Moderna and Oxford/ AstraZeneca vaccines, NVX-CoV2373 is a protein-based vaccine that relies on a well-established, traditional approach and is expected to have a benign safety profile. It can also be stored in refrigerators, a practical advantage that could boost distribution to low- and middle-income countries. The Novavax vaccine also appears to protect against escape by variants. The trial data were collected between January and April 2021, when the Alpha (B.1.1.7) variant, first identified in the United Kingdom, became the main strain circulating in the United States. The vaccine proved to be $93 \%$ effective in preventing symptomatic disease caused by variants of concern circulating during that period. Against symptomatic disease caused by the Beta mutation, an earlier study conducted in South Africa revealed a lower 51\% efficacy among HIV-negative participants. It is unknown whether it can protect against the Delta variant, first identified in India, as that was unlikely to be circulating during the study period. NVX-CoV2373 is a MatrixM-adjuvanted recombinant nanoparticle vaccine engineered from the spike protein genetic sequence of the original SARS-CoV-2 strain. Phase 3 results were announced in a company press release and have yet to be published in a peer-reviewed journal. At the same time, another highly anticipated COVID-19 shot, CureVac's mRNA vaccine $\mathrm{CVnCov}$, failed to meet preset success criteria, delivering only $47 \%$ efficacy against symptomatic disease in a phase $2 / 3$ trial, according to an interim analysis released by the company. CureVac says there were at least 13 variants circulating in the study population during the analysis, which may have reduced the vaccine's efficacy. The German biotech still plans to file for European approval.

Published online: 13 July 2021

https://doi.org/10.1038/s41587-021-00991-8 or applying some combination of these engineering strategies.

To stay relevant, all $\mathrm{mAb}$ developers need to account for the wildcard that is viral evolution, says Jane Osbourn, CSO of Alchemab and former head of the UK BioIndustry Association's antibody taskforce on COVID-19. "A number of the clinical candidates out there are falling over against the [emerging] variants," she says. "So, as a community, we should really be taking the time to think through how you stay ahead of the game in terms of that mutational drift."

In lab studies, sotrovimab seems to maintain its neutralization capacity against all circulating variants of concern, including some of the most worrying versions of the virus, first identified in South Africa, Brazil and India. Several of the leading phase $3 \mathrm{mAb}$ candidates-including Adagio's ADG20, AstraZeneca's AZD7442 and Brii Biosciences' BRII-196 and BRII-198-do as well. But Eli Lilly's two-mAb cocktail is hobbled by escape mutations found in these variants, as is one of the agents, casirivimab, in Regeneron's mAb combination. The other Regeneron agent, imdevimab, retains its activity, in large part because the $\mathrm{mAb}$ targets an epitope that does not overlap with that of its cocktail companion.

That non-redundancy offers some degree of protection against variant-mediated resistance, says Aeron Hurt, principal global medical science director for influenza and COVID-19 therapeutics at Roche, which partnered with Regeneron to handle manufacturing and distribution outside of the United States. "Single antibodies are vulnerable to single mutations," Hurt sayswhereas a cocktail of antibodies that bind at discrete sites provides "an extra insurance policy."

But an even better variant evasion tactic, asserts Adagio CSO Laura Walker, is what her company and Vir have done: both organizations independently screened for ultra-rare broadly neutralizing mAbs that recognize highly conserved epitopes found across the entire family of SARS-like coronaviruses.

The scarcity of these antibodies limits the evolutionary selection pressure for escape mutations in nature, Walker points out. And because conserved residues typically serve essential protein functions, "the virus often can't mutate those residues without suffering a fitness cost," she says, "which means the barrier to escape is typically higher for these broadly neutralizing antibodies."

"But antibodies are not merely things that bind to and neutralize a viral protein," notes Vir CSO Skip Virgin. Through their Fc domain, mAbs also induce innate and adaptive immune responses that help destroy infected cells-and those Fc-mediated activities, Virgin says, "are fundamentally important for treatment of SARS and COVID-19."

Mouse studies published in Cell, in the Journal of Experimental Medicine and as a preprint in recent months now support this idea. But last year, as the COVID-19 mAb race was just heating up, many companiesincluding AstraZeneca, Eli Lilly, Abpro and others-chose instead to dial down effector functions in their mAbs. They wanted to minimize the risk of antibody-dependent enhancement of viral infection, a phenomenon in which virus-specific antibodies can promote, rather than inhibit, disease.

This can be a real problem with certain pathogens, including the respiratory syncytial virus and the dengue virus, but Virgin and his colleagues realized early on that it did not seem to be an issue with SARS-CoV-2. So Vir doubled down on the need for strong Fc receptor binding. Not only did the company leave the effector functions intact for sotrovimab, but it also engineered its successor, the follow-on mAb VIR-7832, to have even greater Fc binding activity.

"The concept is to make the antibody vaccinal," explains Virgin. "We're trying to make the antibody so it not only protects the individual, but it also generates an immune response that outlasts it" through the generation of pathogen-specific $\mathrm{CD}^{+}$and $\mathrm{CD}^{+} \mathrm{T}$ cells responses. The Vir team joined forces last year with Jeffrey Ravetch's lab at Rockefeller University in New York City to demonstrate the Fc engineering concept with an anti-influenza $\mathrm{mAb}$ tested in mice.

\section{"The concept is to make the antibody vaccinal."}

"These are predicted benefits. They haven't been observed in people," Virgin acknowledges, "but that's why we're taking the antibody forward." VIR-7832 and sotrovimab-both of which possess an Fc mutation that confers extended half-life and enhances drug distribution to the lungs-are now part of a master protocol study taking place in the United Kingdom.

Adagio, for its part, has focused its engineering efforts largely on affinity optimization rather than Fc modifications. Although ADG20, like many other next-generation mAbs, does contain $\mathrm{Fc}$ changes that improve its circulation half-life, its most distinguishing selling point is the combination of potency and breadth that it offers.

"We took something from nature that was broad, but not very potent, and then improved the potency while maintaining the 
breadth to essentially make a molecule that would never arise in nature," explains Walker.

Walker and her colleagues started with a natural mAb made in response to a 2003 SARS infection. They then used yeast display technologies to introduce diversity into the parent clone, ultimately finding an offshoot with a handful of genetic changes that gave it 500-fold greater binding affinity to the SARS-CoV-2 spike protein and a 70-fold improvement in neutralization capacitywith no compromise in its breadth of activity across other SARS-related coronaviruses.

In phase 1 testing, clinicians found that a single dose of ADG20 yielded a similar level of virus-neutralizing activity in the blood as that seen among people vaccinated against COVID-19 with one of the marketed mRNA-based products. Pharmacokinetic profiling also indicated that the protection might last for up to a year. Two large global trials are ongoing.

ADG20, like some other late-stage candidates such as AZD7442, also offers the convenience of intramuscular injections-a formulation advantage that Janice Reichert, executive director of the non-profit Antibody Society, argues can be just as important as innovative $\mathrm{mAb}$ designs in the throes of a pandemic or other public health crisis. "Having both would be best, of course," she adds. All the mAbs that already have EUAs must be given intravenously, although versions formulated for intramuscular or subcutaneous administration are now in clinical testing.

Some companies are beginning to explore inhaled delivery as well. Boehringer Ingelheim, for example, is evaluating both infused and inhaled versions of its phase $2 \mathrm{mAb}$ before deciding which administration route to advance into pivotal testing. On 3 June, scientists from IGM Biosciences described promising mouse data on their nasally spritzed product, the immunoglobulin M mAb IGM-6286. And earlier this year, Leyden Labs launched with $€ 40$ million ( $\$ 49$ million) to support a portfolio of intranasal products that protect against respiratory viruses such as influenza and SARS-CoV-2.

According to Dinko Valerio, co-founder and executive chairman of Leyden Labs, the company's nasal spray is designed to thwart viral replication at the site of infection. And because of its localized delivery, patients can get away with much lower doses than they would otherwise need for any systemically administered agent. "If you put it in the nose," Valerio says, "you just need a tiny bit" of the product.

Whatever the mechanism of $\mathrm{mAb}$ delivery, the fact that so much development and therapeutic experimentation is happening today in response to COVID-19 should improve preparedness for the future. Not only are there now products like sotrovimab on the market that offer broad protection against a viral clade that could easily seed the next outbreak, but the engineering approaches being tried now to combat coronaviruses should help inform best practices for creating mAbs designed to thwart other viral families.

"It's good for public health that all these strategies are being tested," remarks James Crowe, director of the Vanderbilt Vaccine Center in Nashville, Tennessee. (A co-founder of IDBiologics, Crowe helped discover the mAbs that form the basis of two cocktail therapies now in clinical testing, AZD7442 from AstraZeneca and another one from Resilience with support from the US Department of Defense.) "It's very exciting scientifically that COVID has allowed this many manufacturers to participate," he says. "Every target is different, but you can typically learn some general principles."

\section{Elie Dolgin \\ Somerville, MA, USA}

Published online: 22 June 2021

https://doi.org/10.1038/s41587-021-00980-x

\section{First Parkinson's patients dosed with dopaminergic neurons}

A clinical study launched in June will be the first to test whether transplanted dopamine-producing neurons can restore brain regions and motor function lost to Parkinson's disease. BlueRock Therapeutics, a company wholly owned by Bayer AG, initiated the phase 1 study to test DA01 cells, dopaminergic neurons derived from human embryonic stem cells, by surgically transplanting them into the putamen, the brain area affected by Parkinson's disease. The aim is for the DA01 cells to replace the lost dopaminergic neurons, stopping and reversing the degenerative process. The trial will assess safety and tolerability of the stem-cell-derived therapy after one year, as well as the transplant's survival and any benefits to motor control. Eventually, BlueRock's commercial product will likely be derived from human induced pluripotent stem cells because such cells are easier to standardize.

Neuroscientist Lorenz Studer and colleagues at Memorial Sloan Kettering Cancer Center developed the protocol for differentiating pluripotent cells into dopaminergic neuron-like cells. Studer is a co-founder of the New York-based BlueRock, which launched in 2016 and was acquired three years later by Bayer for $\$ 1$ billion in up-front and milestone payments. In parallel, and as part of its 'cell + gene' strategy, Bayer is taking a gene therapy program for treating Parkinson's disease into the clinic through its wholly owned subsidiary Asklepios BioPharmaceutical (AskBio). AskBio was founded in 2001 by Jude Samulsky and Sheila Mikhail. The phase 1 trial is testing an adeno-associated virus serotype- 2 (AAV-2) to deliver human glial cell line-derived neurotrophic factor (GDNF) genes to neurons in the putamen to promote regeneration of midbrain neurons. BlueRock is also working to develop futuristic gene-circuit-engineered cell therapies with Senti Biosciences. On 26 May, the companies announced a collaboration to design gene circuits with Senti's Smart Sensors and Regulator Dials to detect and distinguish between a diseased and a healthy cellular environment and respond by fine tuning the therapeutic activity from a cell or gene therapy product, even inside the patient.

Published online: 13 July 2021

https://doi.org/10.1038/s41587-021-00992-7 